\title{
PERFIL E CAPACIDADE FUNCIONAL DOS IDOSOS RIBEIRINHOS ACOLHIDOS NAS CASAS DE APOIO E CENTRO DE CONVIVÊNCIA DO MUNICÍPIO DE ALTAMIRA - PA
}

\section{ARTIGO ORIGINAL}

SIGNORI, Lorinete Trevisan ${ }^{1}$

SIGNORI, Lorinete Trevisan. Perfil e capacidade funcional dos idosos ribeirinhos acolhidos nas casas de apoio e centro de convivência do Município de Altamira - PA. Revista Científica Multidisciplinar Núcleo do Conhecimento. Ano 05, Ed. 05, Vol. 05, pp. 97-114. Maio de 2020. ISSN: 2448-0959, Link de acesso: https://www.nucleodoconhecimento.com.br/saude/idosos-ribeirinhos

\section{RESUMO}

Objetivo: Avaliar a capacidade funcional e o perfil dos idosos ribeirinhos alojados nas casas de apoio e centro de convivência do município de Altamira (PA). Método: Estudo classificado como de campo, descritivo, transversal, com abordagem quantitativa e tratamento estatístico. Amostra formada por 103 (cento e três) idosos. Todos apresentavam a capacidade cognitiva preservada, os quais responderam um questionário com dados epidemiológicos, funcionais e sociodemográficas. Foi utilizado a Escala de Lawton e Brody, o Índice de Katz, e Short Physical Performance Battery (SPPB) para a avaliação das capacidades funcionais. A estatística descritiva simples foi empregada para melhor apresentar os dados epidemiológicos bem como os dados sociodemográficos. Os testes de correlação de Spearman e o Qui-quadrado foram aplicados. Resultados: em relação às atividades básicas de vida diária (ABVD)

1 Mestre em Gerontologia pela universidad Del Atlantico Espanha. Especialista em: Gestão da Clínica nas Regiões da Saúde pelo Sírio Libanês, Traumatologia e ortopedia Universidade do Contestado Unc-Concórdia-SC, Enfermagem do Trabalho FIJ- RJ , Discente em Docência do Ensino Superior UOPAR Universidade do Norte do PR, Enfermeira de Formação Universidade do Contestado UNC, Concórdia- SC. 
foi percebido que $40,8 \%$ dos entrevistados apresentaram dependência parcial. Outros $2,9 \%$ apresentaram dependência total. Quanto às atividades instrumentais de vida diária (AIVD), foi observado que 97,1\% apresentavam dependência parcial e 1,9\% apresentavam dependência total. Por fim, quanto à mobilidade funcional, $24,3 \%$ eram dependentes. A capacidade funcional foi associada a utilização de três medicamentos, ao uso de dispositivos auxiliares ou de marcha, ao consumo de álcool, à utilização de prótese no joelho ou quadril, a artropatias e osteoporose, a diabetes, e, mais significativamente, com relação ao medo de cair. O temor da queda foi a única variável que se relacionou à mobilidade funcional, à AIVD e ABVD. Conclusão: Ficou evidenciado que a variável que mais apresentou associação significativa em relação a capacidade funcional foi o medo de queda. Ficou demonstrado também que ocorreu baixo desempenho na mobilidade funcional, dependência para as AIVD e independência significativa para a realização das ABVD.

Palavras-chave: Idoso, capacidade funcional, casa de apoio.

\section{INTRODUÇÃO}

O crescimento populacional da terceira idade é um fenômeno mundial. Seu crescimento é exponencial e já está consolidado na literatura. Chama atenção o aumento percentual dos idosos longevos (aqueles com 80 anos ou mais), os quais compõem a faixa etária com maior aumento nos últimos tempos ${ }^{1,2}$. Os idosos longevos já ultrapassaram a percentagem anual de crescimento da população de idosos (com 60 anos ou mais). A perspectiva é de que no ano de 2050 o Brasil transpasse o patamar de 13 milhões de idosos longevos, confirmando assim, o envelhecimento da população ${ }^{3}$.

Em virtude desse envelhecimento populacional, um novo panorama epidemiológico se apresenta, caracterizando-se por diversas doenças crônicas e suas complicações. Desta forma, a elevação da expectativa de vida e por conseguinte da população de longevos foi concomitante ao avanço da quantidade das doenças crônicas não transmissíveis (DCNT) e das incapacidades, fazendo com que o perfil epidemiológico 
dos idosos se torne particular e com potencial risco de comprometer a capacidade funcional dessa população ${ }^{4}$.

Com relação a capacidade funcional, os autores Lenardt e Carneiro ${ }^{5}$ explicitam que no indivíduo idoso esta "pode ser entendida como a habilidade de executar, com autonomia e independência, as atividades cotidianas, mantendo o cuidado de si bem como as atividades sociais".

Na população idosa, além da integração social, a capacidade funcional pode ser vista como um significativo indicador de qualidade de vida e saúde, de forma que sua perda ou declínio é fator de risco para mortalidade ${ }^{5,6}$. Em idosos longevos, mesmo com um envelhecimento saudável, alguma redução funcional é esperada, o que torna primordial a identificação precoce dos fatores de riscos, bem como a prevenção do declínio funcional ${ }^{7,8}$.

Avaliar na pessoa idosa a capacidade funcional de forma precoce é de extrema importância, pois permitirá um melhor planejamento das intervenções que favorecerão a manutenção e a potencialização da independência desta população. Dessa forma, é fundamental a compreensão de todos os profissionais da saúde (principalmente os gestores) da funcionalidade e das características que, de certo modo, intervêm na saúde do idoso 5,9 .

Assim, a realização de um estudo a respeito da capacidade funcional e as suas singularidades, se mostra necessário para possibilitar o retrato fidedigno da condição de saúde dos idosos, igualmente para a melhor compreensão de como essas pessoas têm vivido frente a longevidade.

Os entendimentos desses aspectos norteiam ações direcionadas para a população idosa, pois políticas adequadas são elaboradas com base em informações a respeito do público à qual se destinam. Entretanto, pesquisas direcionadas à idosos com foco nas suas condições de saúde - em especial com idosos longevos - são raras e, por essa razão, a temática é pouco difundida ${ }^{8,10}$. 
Esta pesquisa, além de traçar um perfil e avaliar a capacidade funcional dos idosos, pretende suprir o gestor público de informações capazes de melhor orientar na implantação de políticas públicas, visando melhor atender este segmento vulnerável da sociedade. Com a gestão democrática das políticas públicas é possível uma melhor qualidade de resposta em saúde, assegurando que as ações e programas, métodos e modelos, estejam em conformidade com os critérios de qualidade adotados pelo Estado e aprovado pelas comunidades e grupos de usuários. Garantindo, assim, os princípios de disponibilidade, adaptabilidade e aceitabilidade.

Desta forma, a presente pesquisa objetiva analisar o perfil e a capacidade funcional dos idosos ribeirinhos alocados nas casas de apoio e centro de convivência do município de Altamira (PA), região norte do Brasil.

\section{MÉTODO}

Trata-se de um estudo de campo do tipo descritivo, transversal, com abordagem quantitativa e tratamento estatístico com os idosos ribeirinhos das casas de apoio e centro de convivência da cidade de Altamira (PA), região norte do Brasil, totalizando uma amostra de 103 idosos.

Os critérios de inclusão aplicados foram: possuir entre 60 e 81 anos ou mais e apresentar a capacidade cognitiva preservada, em concordância com o Mini Exame do Estado Mental (MEEM), cuja pontuação mínima $\geq 17^{11}$. Foram excluídos os idosos acamados, cadeirantes ou que estavam fora das instituições no momento da coleta dos dados.

A coleta dos dados da pesquisa foi realizada entre os meses de fevereiro e abril do ano de 2018, nos períodos manhã e tarde. Os horários privativos dos idosos nas instituições foram respeitados e as entrevistas foram realizadas em ambiente com iluminação adequada e piso regular.

Para avaliação dos dados epidemiológicos e sociodemográficos foi utilizado um recorte do questionário elaborado pela Rede de Estudos sobre Fragilidade em Idosos 
Brasileiros (Rede FIBRA)12,13. O questionário FIBRA é composto de perguntas objetivas, respondidas por meio do autorrelato, assim como na Pesquisa Nacional por Amostra de Domicílios (PNAD) e nos estudos populacionais Saúde, Bem-Estar e Envelhecimento (SABE) ${ }^{3,14}$.

Para avaliação das DCNT, foi perguntado aos participantes se, no ano anterior, algum médico havia dito que eram portadores de diabetes, pneumopatias, alguma cardiopatia, hipertensão arterial, artropatias, doença cerebrovascular, osteoporose, tumor/câncer e depressão. Quando os entrevistados demonstravam dúvida com relação a alguma questão perguntada, as repostas eram conferidas na ficha de caracterização clínica e social da família, juntamente ao ACS.

Foi acrescentado ao questionário FIBRA, para a ampliação da caracterização epidemiológica, questões sobre a prática regular de atividade física (com ocorrência mínima de 150 minutos por semana), uso de dispositivos auxiliares de marcha, colocação de próteses (artroplastia total/parcial de quadril ou joelho) e sobre medo de cair. Foi avaliado através da Escala Internacional de Eficácia de Quedas (FES-I), validada e adaptada para a população de idosos brasileiros ${ }^{15,16}$.

Para aplicação da FES-I, os participantes respondiam às perguntas sobre o nível de preocupação que possuíam com a possibilidade de queda ao realizarem 16 atividades. A pesquisadora atribuía um valor para cada resposta do participante: 1) não estou preocupado, 2) pouco preocupado, 3) moderadamente preocupado e 4) muito preocupado. Em seguida, os idosos foram classificados em duas categorias: "baixa preocupação com a ocorrência de quedas" ( $\leq 22$ pontos) e "alta preocupação com a ocorrência de quedas" (> 22 pontos). A escala utiliza o termo "preocupação sobre as quedas" porque está intensamente relacionado ao medo, entretanto menos emocional e socialmente aceito pelas pessoas idosas ${ }^{16}$.

Quanto a capacidade funcional, esta foi avaliada por meio do Índice de Katz e da Escala de Lawton e Brody ${ }^{17,18}$. Investigou- se a capacidade dos idosos em executar, respectivamente, seis ABVD (banho, vestir-se, uso do vaso sanitário, transferência, 
continência e alimentação) e sete AIVD (uso do telefone, viagens, compras, preparo de refeições, trabalho doméstico, controle de medicações e do dinheiro).

Para pontuação na escala de Katz, os entrevistados recebiam "1" nas atividades que fossem independentes, ou "0" nas que dependessem de auxílio para sua realização. $\mathrm{Na}$ escala de Lawton e Brody, para cada questão, o participante recebia a seguinte pontuação: 1) não é capaz de realizar a atividade, 2) realiza com auxílio, ou 3) realiza sozinho. Foram considerados "independentes" os participantes que executavam todas as atividades sem auxílio, "dependentes parciais" aqueles que necessitavam de auxílio ou não realizavam pelo menos uma atividade e "dependentes totais" quem necessitava de auxílio em todas as atividades ou não executava nenhuma ${ }^{8,19}$.

O Short Physical Performance Battery (SPPB) foi utilizado para avaliar a capacidade funcional de forma objetiva, por meio de três testes de desempenho. Para avaliação do equilíbrio estático, o entrevistado devia permanecer em pé, sem utilizar bengala ou andador, em três posições diferentes, durante 10 segundos em cada posição. A velocidade de marcha foi avaliada em um percurso de 3 metros. Ao aviso da pesquisadora, o entrevistado iniciava a marcha a partir do ponto inicial marcado até ultrapassar completamente a marca final, andando em passo habitual. Se o entrevistado utilizasse algum dispositivo auxiliar de marcha, poderia usá-lo durante o teste. Por fim, foi avaliada a força muscular dos membros inferiores. O entrevistado iniciava o teste sentado em uma cadeira de $45 \mathrm{~cm}$ de altura, sem encosto para os braços, com os pés apoiados no chão. O entrevistado deveria cruzar os braços sobre o peito, levantar-se da cadeira e sentar-se cinco vezes consecutivas, o mais rápido possível, sem parar entre as repetições. O tempo de cada teste foi marcado e registrado pela pesquisadora por meio de um cronômetro digital ${ }^{20}$.

A pontuação para cada um dos três testes variava numa escala de 0 (pior desempenho) a 4 (melhor desempenho). Os participantes foram classificados em "dependência ou desempenho muito ruim" de 0 a 3 pontos; "baixo desempenho" de 4 a 6 pontos; "moderado desempenho" de 7 a 9 pontos e "bom desempenho" de 10 a 12 pontos $^{20}$. 
Esse estudo foi desenvolvido em consonância com a Resolução no 466/2012 do Conselho Nacional de Saúde (CNS) e aprovado pelo Comitê de Ética em Pesquisa (CEP) da Universidad Europea del Atlántico. Após serem informados sobre os procedimentos e os aspectos éticos da pesquisa, os participantes assinaram o Termo de Consentimento Livre Esclarecido (TCLE).

\section{RESULTADOS}

Dos 103 (cento e três) participantes do estudo 63,1\% da amostra era composta do sexo feminino. Quanto a faixa etária, $86,4 \%$ da amostra possuíam idades entre 60 a 81 anos. A maioria dos indivíduos eram de cor parda $(37,8 \%)$, analfabetos $(62,1 \%)$ e viúvos $(59,2 \%)$. No tocante a condição financeira, os aposentados representaram $92,2 \%$ da amostragem e os que declararam residir com filhos, genrolnora ou netos, representaram $82,5 \%$ do total de idosos pesquisados (Tabela 1 ).

Tabela 1. Caracterização Sociodemográfica dos idosos cadastrados em Altamira, PA, 2018.

\begin{tabular}{|l|l|l|}
\hline Variáveis & N & $\%$ \\
\hline Idade (anos) & & \\
\hline $\mathbf{6 0}$ a 81 & 89 & 86,4 \\
\hline $\mathbf{8 1}$ a 90 & 13 & 12,6 \\
\hline $\mathbf{9 1}$ ou mais & 1 & 1 \\
\hline Sexo & & \\
\hline $\mathbf{M}$ & 38 & 36,9 \\
\hline $\mathbf{F}$ & 65 & 63,1 \\
\hline Cor/Etnia & & \\
\hline Branca & 29 & 28,2 \\
\hline Negra & 35 & 34,0 \\
\hline Parda & 39 & 37,8 \\
\hline Escolaridade & & \\
\hline Analfabeto & 64 & 62,1 \\
\hline
\end{tabular}




\begin{tabular}{|c|c|c|}
\hline 1 a 3 anos & 18 & 17,5 \\
\hline 4 a 7 anos & 21 & 20,4 \\
\hline \multicolumn{3}{|l|}{ Estado Civil } \\
\hline Casado & 33 & 32,0 \\
\hline Solteiro & 2 & 1,9 \\
\hline Viúvo & 61 & 59,2 \\
\hline Divorciado & 7 & 6,8 \\
\hline \multicolumn{3}{|l|}{ Fonte de renda } \\
\hline Aposentadoria & 95 & 92,2 \\
\hline 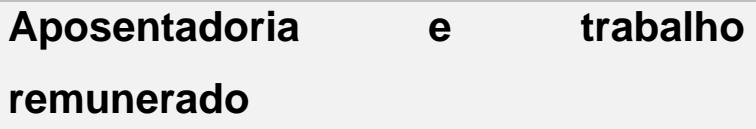 & 5 & 4,9 \\
\hline Trabalho remunerado & 2 & 1,9 \\
\hline Não tem fonte de renda & 1 & 1,0 \\
\hline \multicolumn{3}{|l|}{ Arranjo Domiciliar } \\
\hline Reside sozinho & 8 & 7,8 \\
\hline Com o cônjuge & 7 & 6,8 \\
\hline Com filhos, genrolnora ou netos & 85 & 82,5 \\
\hline Outros arranjos & 3 & 2,9 \\
\hline
\end{tabular}

No tocante a pesquisa epidemiológica, foi verificado que, do total de idosos avaliados, $6,8 \%$ relataram não ter DCNT. A hipertensão foi apontada como e enfermidade predominante, estando presente em $80,6 \%$ da amostra, seguida da artropatia, com $56 \%$. O medo de cair é preocupante para todos os idosos, mas para $54,4 \%$ relataram alta preocupação. Foi verificado, ainda, que $96,1 \%$ dos entrevistados não utiliza nenhum tipo de prótese e 78,6\% não necessita de dispositivos auxiliares de marcha, entretanto $67 \%$ necessitam três ou mais tipos de medicação por dia. O tabagismo atinge $9,7 \%$ e o consumo de álcool (esporádico) é praticado por $5,8 \%$. Por fim, $84,5 \%$ dos entrevistados relataram não praticar exercício físico (Tabela 2). 
Tabela 2. Características epidemiológicas dos idosos cadastrados em Altamira, PA, 2018.

\begin{tabular}{|c|c|c|}
\hline Variáveis & $\mathbf{N}$ & $\%$ \\
\hline \multicolumn{3}{|l|}{ Doenças autorreferidas } \\
\hline Cardiopatias* & 12 & 11,7 \\
\hline Hipertensão & 83 & 80,6 \\
\hline AVE & 17 & 16,5 \\
\hline Diabetes & 28 & 27,2 \\
\hline Câncer & 9 & 8,7 \\
\hline Artropatias & 58 & 56,3 \\
\hline Doença Pulmonar & 14 & 13,6 \\
\hline Depressão & 10 & 9,7 \\
\hline Osteoporose & 41 & 39,8 \\
\hline \multicolumn{3}{|l|}{ Medo de Cair (FES-I) } \\
\hline Baixa preocupação & 47 & 45,6 \\
\hline Alta preocupação & 56 & 54,4 \\
\hline \multicolumn{3}{|c|}{$\begin{array}{l}\text { Uso de dispositivos Auxiliares de } \\
\text { Marcha }\end{array}$} \\
\hline Bengala & 19 & 18,5 \\
\hline Andador Fixo & 3 & 2,9 \\
\hline Não faz uso & 81 & 78,6 \\
\hline \multicolumn{3}{|l|}{ Prótese } \\
\hline Prótese Total/Parcial de Quadril & 3 & 2,9 \\
\hline Prótese Total/Parcial de Joelho & 1 & 1,0 \\
\hline Não possui & 99 & 96,1 \\
\hline \multicolumn{3}{|l|}{ Número de medicamentos } \\
\hline 0 & 8 & 7,8 \\
\hline $1-2$ & 26 & 25,2 \\
\hline $3-4$ & 42 & 40,8 \\
\hline 5 ou mais & 27 & 26,2 \\
\hline
\end{tabular}




\begin{tabular}{|l|l|l|}
\hline Queda (últimos $\mathbf{6}$ meses) & \multicolumn{2}{|l|}{} \\
\hline Sim & 34 & 33,0 \\
\hline Não & 69 & 67,0 \\
\hline Número de quedas (últimos 6 meses) & & \\
\hline $\mathbf{1}$ & 16 & 15,6 \\
\hline $\mathbf{2}$ & 9 & 8,7 \\
\hline $\mathbf{3}$ ou mais & 9 & 8,7 \\
\hline Tabagismo & & \\
\hline Sim & 10 & 9,7 \\
\hline Fumou mas parou & 61 & 59,2 \\
\hline Nunca fumou & 32 & 31,1 \\
\hline Consumo de Bebida Alcoólica & & \\
\hline Sempre & 0 & 0 \\
\hline Às vezes & 4 & 3,9 \\
\hline Raramente & 2 & 1,9 \\
\hline Nunca & 97 & 94,2 \\
\hline Exercício Físico & & \\
\hline Sim & 16 & 15,5 \\
\hline Não & 87 & 84,5 \\
\hline
\end{tabular}

Em relação às Atividades Básicas da Vida Diária (ABVD), a pesquisa demonstra que $56,3 \%$ dos idosos questionados declararam ser independentes e nas Atividades Instrumentais da Vida Diária (AIVD), 97,1\% que declararam dependência parcial. Por fim, quando avaliado o quesito da mobilidade funcional, 33,0\% apresentaram baixo desempenho (Tabela 3).

Tabela 3. Classificação da capacidade funcional, segundo as ABVD (Atividade Básica de Vida Diária), AIVD (Atividade Instrumental de Vida Diária) e mobilidade funcional dos idosos cadastrados em Altamira, PA, 2018.

\begin{tabular}{|l|l|l|}
\hline Variáveis & N & $\%$ \\
\hline Atividades Básicas de Vida Diária & & \\
\hline
\end{tabular}




\begin{tabular}{|l|l|l|}
\hline Independência & 58 & 56,3 \\
\hline Dependência Parcial & 42 & 40,8 \\
\hline Dependência Total & 3 & 2,9 \\
\hline $\begin{array}{l}\text { Atividades Instrumentais de Vida } \\
\text { Diária }\end{array}$ & & \\
\hline Independência & 1 & 1,0 \\
\hline Dependência Parcial & 100 & 97,1 \\
\hline Dependência Total & 2 & 1,9 \\
\hline Mobilidade Funcional & & 10,7 \\
\hline Bom desempenho & 11 & 32,0 \\
\hline Moderado Desempenho & 33 & 33,0 \\
\hline Baixo Desempenho & 34 & 24,3 \\
\hline Dependência & 25 & \\
\hline
\end{tabular}

As características epidemiológicas significativamente associadas à dependência para $A B V D$ e AIVD e à mobilidade funcional foram submetidas a um teste de correlação estatística, verificando que existe relação entre elas.

Como resultado, verificou-se que nas $A B V D$ foi associada ao uso de dispositivos auxiliares de marcha $(\rho=0,275, p \leq 0,01)$, colocação de prótese no quadril ou joelho ( $\rho$ $=0,214, p \leq 0,05)$, uso de 3 ou mais medicamentos $(\rho=0,234, p \leq 0,05)$, artropatias ( $\rho$ $=0,235, p \leq 0,05)$, osteoporose $(\rho=0,265, p \leq 0,01)$, consumo moderado de bebida alcoólica $(\rho=-0,217, p \leq 0,05)$ e, mais significativamente, ao medo de cair $(\rho=0,387$, $p \leq 0,01)$ (Tabela 4).

Tabela 4. Características epidemiológicas significativamente associadas à dependência para ABVD e AIVD e à mobilidade funcional segundo o Teste de Correlação de Spearman em idosos de Altamira, PA, 2018.

\section{Coeficientes de correlação de Spearman ( $\rho$ )}

\begin{tabular}{|l|l|l|l|}
\hline & ABVD & AIVD & Mobilidade Funcional \\
\hline Variáveis & & & \\
\hline
\end{tabular}




\begin{tabular}{|c|c|c|c|}
\hline Diabetes & - & - & $-0,211 p=0,023^{*}$ \\
\hline Artropatias & $\begin{array}{l}0,235 \\
p=0,018^{*}\end{array}$ & - & - \\
\hline Osteoporose & $\begin{array}{l}0,265 \\
p=0,007^{* *}\end{array}$ & - & $\begin{array}{l}-0,388 \neq \\
p=0,000^{\star *}\end{array}$ \\
\hline Medo de Cair (FES-I) & $\begin{array}{l}0,387 \neq \\
p=0,000^{* *}\end{array}$ & $\begin{array}{l}0,237 \\
p=0,016^{*}\end{array}$ & $\begin{array}{l}-0,521 \\
p=0,000^{* *}\end{array}$ \\
\hline $\begin{array}{l}\text { Dispositivo auxiliares } \\
\text { de marcha }\end{array}$ & $\begin{array}{l}0,275 \\
p=0,005^{\star *}\end{array}$ & - & $\begin{array}{l}-0,471 \neq \\
p=0,000^{\star *}\end{array}$ \\
\hline $\begin{array}{l}\text { Prótese de quadril ou } \\
\text { joelho }\end{array}$ & $\begin{array}{l}0,214 \\
p=0,030^{*}\end{array}$ & - & - \\
\hline $\begin{array}{l}\text { Uso de } 3 \text { ou mais } \\
\text { medicamentos }\end{array}$ & $\begin{array}{l}0,234 \\
p=0,018^{*}\end{array}$ & & $\begin{array}{l}-0,297 \\
p=0,002^{\star *}\end{array}$ \\
\hline $\begin{array}{l}\text { Consumo moderado de } \\
\text { bebida alcoólica }\end{array}$ & $\begin{array}{l}-0,217 \\
p=0,028^{\star}\end{array}$ & $\begin{array}{l}-0,261 \\
p=0,008^{* *}\end{array}$ & - \\
\hline
\end{tabular}

Nota: $\rho=$ Coeficiente de Correlação de Spearman; **.p $\leq 0,01 ;{ }^{*} . p \leq 0,05$; \#.Significância no Qui-quadrado. 


\section{DISCUSSÃO}

No presente estudo, foi verificado maior quantidade de indivíduos do sexo feminino. Esse predomínio das mulheres idosas também foi descrito em outros estudos similares realizados de outras cidades do Brasil, como no estudo de Matos et al. ${ }^{19}$. Esta maior representatividade do sexo feminino na terceira idade é um fenômeno mundial, sendo também chamado de feminização da velhice, como bem observado nos estudos realizados por Almeida et al. ${ }^{21}$.

A maior parte da amostragem da pesquisa declarou residir com filhos, genros/nora ou netos. Este resultado se aproxima de outros estudos com idosos realizados no Brasil, o que, de acordo com Perseguino et al. ${ }^{22}$, pode ser uma característica dos idosos em nosso país. Diferentemente do constatado neste estudo, nos países da Europa e da América do Norte, os idosos têm por hábito viver sozinhos, evidenciando as diferenças culturais entre os países quanto ao arranjo domiciliar e familiar dos idosos.

A questão da dependência é comumente abordada nos estudos científicos e ocorre com significativa frequência nos estágios avançados da velhice. A terminologia 'idoso funcional' decorre de um programa de avaliação de idosos implantado pela Organização Mundial da Saúde, órgão das Nações Unidas, objetivando estudar as limitações e dificuldades na realização de atividades cotidianas ${ }^{23}$.

No presente estudo, os idosos foram, em sua maioria, independentes na realização das ABVD, resultado que corrobora os estudos realizados por Brito et al. ${ }^{8}$.

Quanto às AIVD, 99\% dos participantes mostrou algum grau de dependência. Esse dado corrobora com o estudo realizado por Freitas et al. ${ }^{24}$, que avaliou a capacidade funcional de idosos ( $\geq 60$ anos) pelo Índice de Katz e pela Escala de Lawton e Brody. O estudo apontou associação significativa entre dependência nas AIVD de idosos. Como apresentado, em virtude de a amostra ser composta por idosos, o elevado índice de dependência nas AIVD já era um resultado esperado. 
A presente pesquisa confirma os achados de Menéndez et al. ${ }^{25}$, descritos no seu estudo transversal com idosos ( $\geq 60$ anos) de sete países da América Latina e Caribe (Barbados, Argentina, Cuba, México, Uruguai, Chile e Brasil). O nível de dificuldade para executar as AIVD foi maior do que para as ABVD em todos os países estudados, mesmo sendo sua amostra composta por idosos mais jovens, comparada à amostra deste estudo.

A execução das AIVD envolve a integração de vários sistemas, como o nervoso e o musculoesquelético, sendo que nos idosos, esses sistemas geralmente encontram-se em um estado acentuado de declínio. Essas múltiplas integrações acarretam maior complexidade de execução das AIVD, por isso há uma hierarquia entre as perdas funcionais, sendo a dependência nas AIVD anterior à das ABVD, tanto em idosos mais jovens como em mais velhos ${ }^{26}$.

A capacidade funcional de idosos já foi associada a fatores diferentes dos descritos na presente pesquisa, como fatores sociodemográficos, hospitalização recente, histórico de AVE, quedas e declínio cognitivo $^{12}$. No presente estudo, houve associação entre a capacidade funcional de idosos e o uso de medicamentos, assim como foi igualmente verificado no estudo de Brito et al. ${ }^{8}$.

Apesar dos benefícios da prática da atividade física para a saúde dos idosos serem consistentemente demonstrados na literatura, na presente pesquisa, não se observou associação da capacidade funcional à prática do mesmo.

O estudo de Pahor et al. ${ }^{27}$, realizado nos Estados Unidos, com um grupo de idosos sedentários entre 70 a 89 anos, contrapõe-se aos nossos achados. Os idosos foram inicialmente divididos em dois grupos: um realizava atividades físicas diariamente e o outro não. Os idosos pertencentes ao grupo do programa de atividade física demonstraram aumento na pontuação do SPPB, mostrando aprimoramento da mobilidade funcional.

A atividade física regular é excelente para a manutenção dos aspectos funcionais dos idosos. Comparando-se o exercício físico praticado pelos idosos do exterior com os 
exercícios praticados pelos idosos de Altamira, pode-se inferir que a não associação entre exercício físico e capacidade funcional pode estar ligada ao conceito da expressão "exercício físico". Foi constatado pela amostragem da pesquisa que o idoso de Altamira está acostumado a deslocar diariamente a pé, cumprindo toda uma rotina que inclui pegar transporte público, permanecer em filas, enfrentar ladeiras com sacolas de compras, entre outros obstáculos, realizando verdadeiros exercícios físicos disfarçados em sua rotina do dia a dia.

Quanto às variáveis que apresentaram correlação significativa com a capacidade funcional, o consumo moderado de álcool apresentou associação negativa com a dependência nas ABVD $(\rho=-0,217, p \leq 0,05)$ e AIVD $(\rho=-0,261, p \leq 0,05)$. Estudo realizado na Suécia por Agahi et al. ${ }^{28}$ apresentou resultados semelhantes.

Segundo Agahi et al. ${ }^{28}$, o consumo moderado de álcool, em grupo, favorece o vínculo social e melhora a sensação de bem-estar. Portanto, considerando-se que os idosos da presente amostra apresentavam moderado a baixo consumo de álcool, uma possível explicação para a associação negativa com a dependência seria a melhora do bem-estar, que repercute diretamente na funcionalidade, proporcionando qualidade de vida, como reunião com os amigos, participação na comunidade e socialização, têm associação positiva com as ABVD e AIVD.

Em contrapartida, as consequências nocivas do álcool podem ser mais intensas nos idosos, pois, devido às mudanças fisiológicas características da idade (diminuição da massa magra, da água corporal, e do metabolismo hepático), o nível de álcool no sangue é maior, de modo que seus efeitos são sentidos, mesmo que o consumo não seja em grande quantidade. Sem falar que existe a possibilidade de interação negativa do álcool com os medicamentos proporcionando a exacerbação dos seus efeitos adversos, além de agravamento e descompensação de doenças crônicas, como foi relatado nos estudos de Agahi et al. ${ }^{28}$.

A presença de algumas doenças crônicas, como artropatias e osteoporose, associouse de forma positiva à dependência nas $\operatorname{ABVD}(\rho=0,235, p \leq 0,05)$ e $(\rho=0,265, p \leq$ 0,01 ), respectivamente, e a presença de osteoporose e diabetes associou-se de forma 
negativa à mobilidade funcional $(\rho=-0,211, p \leq 0,05)$ e $(\rho=-0,388, p \leq 0,01)$, respectivamente, portanto idosos que têm essas DCNT parecem estar mais vulneráveis à dependência funcional. Estes dados apresentam semelhança aos resultados obtidos por Nogueira et al. ${ }^{29}$.

Esta associação entre a osteoporose e mobilidade funcional, descrita na presente amostra, também foi apontada no estudo de Santos et al. ${ }^{30}$, que analisou a associação entre massa óssea e capacidade funcional de idosos. Os autores avaliaram a capacidade funcional através dos três testes funcionais que compõem o SPPB e concluíram que idosos do sexo masculino, com melhor desempenho nos testes funcionais, apresentaram maiores valores de conteúdo mineral ósseo de fêmur, portanto, menor risco de osteoporose.

O uso de próteses esteve associado à dependência nas ABVD ( $\rho=0,214, p \leq 0,05)$, sendo um importante fator limitador para os idosos. A insegurança com a colocação e uso de prótese pode se somar a outros aspectos, como declínio das funções físicas e medo de cair, causando ainda mais impacto na funcionalidade. Deste modo, mais estudos devem ser realizados, em amostras particulares de idosos, para esclarecimento dos reais benefícios das próteses e do seu impacto na capacidade funcional.

O uso de dispositivos auxiliares de marcha se associou à dependência nas ABVD ( $\rho=$ $0,275, p \leq 0,01$ ) e apresentou a segunda correlação mais significativa com a mobilidade funcional $(\rho=-0,471, p \leq 0,01)$. Nesta pesquisa, $18,5 \%$ dos idosos utilizavam bengala, assim como na amostra do National Health and Aging Trends Study (NHATS), realizada em 2011 nos Estados Unidos, que apontou a bengala como o dispositivo auxiliar mais usado pelos idosos. De acordo com a entidade avaliadora, a limitação na execução de atividades cotidianas foi significativamente maior entre os que faziam uso de bengala, entretanto os idosos entrevistados alegaram sentir maior segurança ${ }^{31}$.

Para Bateni e Maki32, o uso de dispositivos auxiliares de marcha "pode ser naturalmente um indicador de comprometimento de equilíbrio e declínio funcional". 
Além disso, estes aparelhos exigem dos idosos o "uso de recursos cognitivos, controle postural e coordenação para levantar e avançar com o dispositivo". Essas demandas poderiam gerar um maior grau de dificuldade para a realização de atividades cotidianas. Considerando-se que esses dispositivos são usados para suprir déficits de equilíbrio, faz-se necessário treinamento do usuário por profissionais especializados para garantir a segurança do idoso.

Outro aspecto avaliado na pesquisa diz respeito aos medicamentos. A questão da interação medicamentosa é fator igualmente importante para o estudo da mobilidade funcional. Via de regra, idosos costumam necessitar de diversos tipos de medicamentos ao longo do dia. O uso de três ou mais medicamentos associou-se de forma negativa à mobilidade funcional $(\rho=-0,297, p \leq 0,01)$ e positiva à dependência nas $\operatorname{ABVD}(\rho=0,234, p \leq 0,05)$. Em geral, a população idosa é mais vulnerável ao uso de múltiplos medicamentos, o que implica prejuízo à independência funcional, o que ratifica os achados do presente estudo.

Nos Estado Unidos, estudo capitaneado por Charlesworth et al. ${ }^{33}$ encontrou resultado semelhante aos encontrados nesta pesquisa, dentre os quais destaca-se o percentual de idosos que apresentavam limitações. Do total da amostragem (mais de 13 mil idosos), 26,4\% declararam fazer uso de um a quatro medicamentos ao dia e afirmaram possuir alguma limitação na mobilidade funcional.

O risco de queda da própria altura (medo de cair) foi a única variável que se associou aos três componentes da capacidade (funcional ABVD, AIVD e mobilidade funcional), além de ter apresentado a associação mais significativa com a dependência nas ABVD $(\rho=0,387, p \leq 0,01)$ e, de forma negativa com a mobilidade funcional $(\rho=-0,521$, $p \leq 0,01)$, sendo assim um fator de declínio funcional para idosos.

As consequências de uma queda da própria altura atingem aspectos físicos, funcionais, psicológicos e sociais. Sua frequência aumenta à medida que os indivíduos se tornam mais velhos e está associada ao número de quedas. Mesmo idosos que não experimentaram um evento de queda também relatam medo de cair. 
A relação entre medo de cair e declínio nas atividades de vida diária é tão forte em idosos não caidores quanto em caidores ${ }^{34,35}$.

Na presente amostra, diferentemente do medo de cair, a queda não esteve associada à dependência funcional. Do total de idosos pesquisados, 33\% relataram episódio de queda, enquanto $54,4 \%$ tinham alta preocupação em cair. Este resultado sugere que o medo de cair em idosos é mais nocivo para a independência funcional do que a própria queda, pois limita psicologicamente o indivíduo para as atividades quotidianas.

Segundo Cumming et al. ${ }^{34}$, uma possível hipótese para esse fato é que alguns idosos não caidores podem ter uma percepção das quedas como um evento catastrófico, envolvendo fraturas e hospitalização, enquanto idosos que já caíram têm a ideia de que a maioria das quedas não é nociva, portanto não causa danos físicos.

A questão da funcionalidade do idoso não se encerra na apresentação de dados de pesquisa. Tão importante quanto a coleta de dados é sua análise e posterior interpretação dos resultados. A busca por fatores de risco ou eventos correlatos é extremamente importante para expandir o conhecimento e melhorar a qualidade de vida desta população. Assim, é importante discutir as limitações desta pesquisa, pois, apesar de indicar a presença de correlação de algumas variáveis com a capacidade funcional, o tipo de estudo transversal não permite estabelecer a relação causal entre essas variáveis. Outra limitação é que a amostra não permite a ampliação desses dados para outros perfis de idosos, como os idosos institucionalizados, o que demandaria novos e maiores estudos sobre a temática.

\section{CONCLUSÃO}

Este trabalho objetivou avaliar, através de pesquisa de campo, a capacidade funcional dos idosos que frequentam o sistema assistencial de Altamira, no Pará. A análise do perfil funcional dos idosos permite mapear informações importantes que poderão subsidiar melhores políticas públicas e sociais no futuro. 
Ao longo do estudo foi possível perceber que, em relação à capacidade funcional, os idosos foram, em sua maioria, independentes na realização das $A B V D$, dependentes nas AIVD e apresentaram baixo desempenho na mobilidade funcional. O medo de cair foi a única variável que apresentou associação com a ABVD, AIVD e a mobilidade funcional. O medo de cair representou, também, a associação positiva mais significativa com a dependência nas ABVD e negativa com a mobilidade funcional.

Em relação aos aspectos socioeconômicos, de moradia e as condições de saúde, a maioria dos idosos pesquisados era do sexo feminino e apresentava renda própria, residia com familiares e possuía boas condições gerais de saúde. Outro ponto observado nesta pesquisa é o quanto ainda existe por ser realizado pelos poderes públicos. O público idoso é carente de políticas efetivas por quase todos os aspectos. É sabido que em períodos de crise os recursos faltam justamente nos setores que mais necessitam, pois, muitas famílias passam a necessitar mais dos serviços de amparo e assistência social. Neste sentido, em que pese a legislação ser bastante convincente, a efetividade dos poderes em implementar ações neste sentido é baixíssima.

Como os idosos representam atualmente o segmento populacional que mais cresce em termos proporcionais, ressalta-se a importância de pesquisar e debater, nos âmbitos acadêmico e profissional, as diversas questões relacionadas ao processo de envelhecimento e as condições de funcionalidade dos idosos. Os resultados deste estudo contribuem para elucidar aspectos epidemiológicos que impõem riscos à funcionalidade dos idosos, permitindo estratificá-los quanto ao grau de risco funcional, garantindo acompanhamento e tratamento mais específico. Em razão da importância da temática, sugere-se a realização de futuros estudos para investigar se as associações aqui descritas podem ser ampliadas para idosos de diferentes amostras.

\section{REFERÊNCIAS}

1. UNFPA - Fundo das Nações Unidas para a População. Envelhecimento no século XXI: celebração e desafio. 2012 [acesso em 10 jul. 2018]. Disponível 
em: http://www.unfpa.org/sites/default/ files/ pub-pdf/Portuguese-ExecSummary_0.pdf

2. Brasil. Ministério da Saúde. Atenção à saúde da pessoa idosa e envelhecimento. 2010. [acesso em 12 jun. 2018]. Disponível em: http://bvsms.saude.gov.br/bvs/ publicacoes/abcad19.pdf

3. IBGE - Instituto Brasileiro de Geografia e Estatística. Projeção da população do Brasil por sexo e idade - 1980, 2050. 2008 [acesso em 7 jul. 2018]. Disponível em: http://biblioteca.ibge.gov.br/ visualizacao/livros/liv41229.pdf

4. Porciúncula RCR, Carvalho EF, Barreto KML, Leite VMM. Perfil socioepidemiológico e autonomia de longevos em Recife-PE, Nordeste do Brasil. Revista Brasileira de Geriatria e Gerontologia 2014;17(2):315-25.

5. Lenardt MH, Carneiro NHK. Associação entre as características sociodemográficas e a capacidade funcional de idosos longevos da comunidade. Cogitare enfermagem 2013;18(1): 13-20.

6. Cruz GEC, Ramos LR. Limitações funcionais e incapacidades de idosos com síndrome de imunodeficiência adquirida. Acta Paulista de Enfermagem 2015;28(5):488-93.

7. Imai E, Tsubota-Utsugi M, Kikuya M, Satoh M, Inoue R, Hosaka M et al. Animal protein intake is associated with higher-level functional capacity in elderly adults: the Ohasama study. Journal of the American Geriatrics Society 2014;62(3):426-434.

8. Brito TA, Fernandes MH, Coqueiro RS, Jesus CS, Freitas R. Capacidade funcional e fatores associados em idosos longevos residentes em comunidade: estudo populacional no Nordeste do Brasil. Fisioterapia e Pesquisa 2014;21(4):308-13.

9. Barbosa BR, Almeida JM, Barbosa MR, Rossi-Barbosa LAR. Avaliação da capacidade funcional dos idosos e fatores associados à incapacidade. Ciênc. saúde coletiva 2014; 19(8):3317-3325.

10. Ribeiro DKMN, Lenardt MH, Michel T, Setoguchi LS, Grden CRB, Oliveira ES. Fatores contributivos para a independência funcional de idosos longevos. Rev Esc Enferm USP 2015;49(1):89-95. 
11. Brasil. Ministério da Saúde. Secretaria de Atenção à Saúde. Departamento de Atenção Básica. (2007). Envelhecimento e Saúde da Pessoa Idosa. [acesso em 28 jul. 2018]. Disponível em: //bvsms.saude.gov.br/bvs/publicacoes/abcad19.pdf

12. Perez M, Lourenço RA. Rede FIBRA-RJ: fragilidade e risco de hospitalização em idosos da cidade do Rio de Janeiro, Brasil. Cad. de Saúde Pública 2013;29(7):1381-91.

13. Pinto JM, Neri AL. Doenças crônicas, capacidade funcional, envolvimento social e satisfação em idosos comunitários: Estudo Fibra. Ciência \& Saúde Coletiva 2013;18(12):3449-60.

14. Lebrão ML, Duarte YAO. SABE - Saúde, bem estar e envelhecimento. O projeto SABE no município de São Paulo: uma abordagem inicial. Brasília: Organização Pan-Americana da Saúde; 2003.

15. Garber CE, Blissmer B, Deschenes MR, Franklin BA, Lamonte MJ, Lee IM et al. American College of Sports Medicine position stand. Quantity and quality of exercise for developing and maintaining cardiorespiratory, cardiorespira- tory, musculoskeletal, and neuromotor fitness in apparently healthy adults: guidance for prescribing exercise. Medicine \& Science in Sports \& Exercise 2011;43(7):1334-59.

16. Camargos F, Dias R, Dias J, Freire M. Adaptação transcultural e avaliação das propriedades psicométricas da Falls Efficacy Scale - International em idosos Brasileiros (FES-I-BRASIL). Revista Brasileira de Fisioterapia 2010;14(3):23743.

17. Lino VTS, Pereira SRM, Camacho LAB, Ribeiro Filho ST, Buksman S. Adaptação transcultural da Escala de Independência em Atividades da Vida Diária (Escala de Katz). Cad. Saúde Pública 2008;24(1):103-12.

18. Santos RL, Virtuoso Júnior JS. Confiabilidade da versão brasileira da escala de atividades instrumentais da vida diária. Revista Brasileira em Promoção da Saúde 2008; 21(4):290-6.

19. Matos IE, Carmo CN, Santiago LM, Luz LL. Factors associated with functional incapacity in elders living in long stay institutions in Brazil: a cross- sectional study. BMC Geriatrics 2014;14(47):2-9. 
20. Nakano M M. Versão brasileira da Short Physical Performance Battery - SPPB: adaptação cultural e estudo da confiabilidade [dissertação]. Campinas: Universidade Estadual de Campinas; 2007.

21. Almeida AV, Mafra SCT, Silva EP, Kanso S. A feminização da velhice: em foco as características socioeconômicas, pessoais e familiares das idosas e o riso social. Textos e Contextos 2015;14(1):115-31.

22. Perseguino MG, Horta ALM, Ribeiro CA. A família frente a realidade do idoso de morar sozinho. Rev. Bras. Enferm 2016;70(2):235-41.

23. OMS - Organização Mundial da Saúde. CIF: Classificação Internacional de Funcionalidade, Incapacidade e Saúde. São Paulo: EDUSP; 2004.

24. Freitas RS, Fernandes MH, Coqueiro RS, Reis Júnior WM, Vasconcelos $S$, Brito TA. Capacidade funcional e fatores associados em idosos: estudo populacional. Acta Paulista de Enfermagem 2012;25(6):933-39.

25. Menéndez J, Guevara A, Arcia N, León Díaz EM, Marín C, Alfonso JC. Enfermedades crónicas y limitación funcional en adultos mayores: estudio comparativo en siete ciudades de América Latina y el Caribe. Revista Panamericana de Salud Pública 2005;17(5-6):353-61.

26. Perracini MR, Fló JM, Guerra RO. Funcionalidade e envelhecimento. Rio de Janeiro: Guanabara Koogan; 2009.

27. Pahor M, Blair SN, Espeland M, Fielding R, Gill TM, Guralnik JM, et al. Effects of a physical activity intervention on measures of physical performance: Results of the lifestyle interventions and independence for Elders Pilot (LIFE-P) study. Journals of Gerontology. Series A: Biological Sciences and Medical Sciences 2006;61:1157-65.

28. Agahi N, Kelfve S, Lennartsson C, Kareholt I. Alcohol consumption in very old age and its association with survival: A matter of health and physical function. Drug \& Alcohol Dependence 2016;159:240-45.

29. Nogueira SL, Ribeiro RCL, Rosado LEFPL, Franceschini SCC, Ribeiro AQ, Pereira ET. Fatores determinantes da capacidade funcional em idosos longevos. Brazilian Journal of Physical Therapy 2010;14(4):322-29. 
30. Santos VR, Christofaro DGD, Gomes IC, Codogno JS, Santos LL, Freitas Junior IF. Associação entre massa óssea e capacidade funcional de idosos com 80 anos ou mais. Rev. Bras. Ortop. 2013;48(6):512-18.

31. NHATS - National Health and Aging Trends Study. 2011 [acesso em 10 jul. 2018]. Disponível em: https://www.nhats.org/

32. Bateni H, Maki B. Assistive devices for balance and mobility: benefits, demands, and adverse consequences. Archives of Physical Medicine and Rehabilitation 2005;86:134-45.

33. Charlesworth C, Smit E, Lee D, Alramadhan F, Odden MC Polypharmacy Among Adults Aged 65 Years and Older in the United States: 1988-2010. Journals of Gerontology. Series A: Biological Sciences and Medical Sciences 2015;70(8):989-95.

34. Cumming RG, Salkeld G, Thomas M, Szonyi GJ. Prospective Study of the Impact of Fear of Falling on Activities of Daily Living, SF-36 Scores, and Nursing Home. Journals of Gerontology. Series A: Biological Sciences and Medical Sciences 2000;55(5):299-305.

35. Kumar A, Carpenter H, Morris R, lliffe S, Kendrick D. Which factors are associated with fear of falling incommunity-dwelling older people? Age and Ageing 2013;43:76-84.

Enviado: Março, 2020.

Aprovado: Maio, 2020. 